\title{
Computer Diagnostic and Monitoring Device Based on the Theory of Identification Measurement of Signals
}

\author{
Alexander Kashevkin ${ }^{1, *}$, Yuriy Klikushin ${ }^{2}$, Abay Koshekov ${ }^{1}$, Bibigul Koshekova ${ }^{1}$, Sergey Latypov ${ }^{1}$, Natalya \\ Kalantayevskaya $^{1}$ and Galina Savostina ${ }^{1}$ \\ ${ }^{1}$ Kazakhstan, North-Kazakhstan region, Petropavlovsk, North Kazakhstan State University named after M. Kozybayev \\ ${ }^{2}$ Russian Federation, Omsk region, Omsk, Omsk State Technical University \\ ${ }^{*}$ Corresponding author
}

\begin{abstract}
The research results in the field of construction of intellectual computer devices for increase of reliability of vibrodiagnostics tests results of the industrial equipment are presented in the article. These results can be achieved by applying the theory of identification measurements of signals. The technique of intellectual processing of vibration signals and the technology of creation of a computer device are considered.
\end{abstract}

Keywords-theory of identification signals measurements; expert systems; vibrodiagnostics; computer device

\section{I.INTRODUCTION}

One of the innovative scientific trends in the development of scientific and technological progress is the introduction of intelligent technologies. Leading oil and gas companies have long been working towards "intellectualization" by creating expert systems for monitoring the status and diagnosis of machines and mechanisms. However, the range of solved tasks, in this case, is limited, and the developed intellectual technologies are not effective enough.

This is primarily due to the fact that at the moment the application of expert systems in the diagnosis of equipment allows us to recognize only the states of groups of defects with the same characteristics from the spectral characteristics, the accuracy of identifying defects reaches 0.6 and such systems are highly specialized [1].

It should be noted that it is performed additional analysis of time characteristics, then the accuracy of identifying defects will increase. Currently, there are various technologies digitally processing complex diagnostic signals, allowing for their indepth analysis and requiring the construction of diagnostic and monitoring systems in the structure of artificial intelligence. Examples of such technologies are wavelet transform [2], neural network methods [3], etc.

The authors propose to analyze the time characteristics of complex signals containing stationary and nonstationary components with the help of technologies of the theory of identification signals measurements (TISM) [4], which allows to recognize the waveform of signals with the establishment of logical connections between types of defects and values of estimates of the degree of regularity-randomness of signals.

\section{Methodology And Instruments Of ReseARCH}

The content of the TISM is the measurement of the form the distribution of instantaneous values and variability - the distribution of the time intervals of the signal and its characteristics with the processing of identification parameters in the structure of global standards by means of identification scales [5].

Based on the provisions of the TISM, a method for processing diagnostic signals was developed, algorithmically implemented in the following steps.

Step 1. Determination of the identification number from the samples with the $\mathrm{N}$ volume of the input signal $\mathrm{X}_{1}(t)$ and its increment $\Delta \mathrm{X}_{1}(t)$ according to the formula:

$$
Y_{1}(N)=\frac{\left|\overline{\Delta \mathrm{X}_{1}(t)}\right|}{\left|\overline{\mathrm{X}_{1}(t)}\right|} .
$$

Step 2. Determination of the identification number from the samples with the $\mathrm{N}$ volume of the ranked function $\mathrm{X}_{2}(t)$ of the input signal $\mathrm{X}_{1}(t)$ and its increment $\Delta \mathrm{X}_{2}(t)$ according to the formula:

$$
\mathrm{Y}_{2}(\mathrm{~N})=\frac{\left|\overline{\Delta \mathrm{X}_{2}(\mathrm{t})}\right|}{\left|\overline{\mathrm{X}_{2}(\mathrm{t})}\right|}
$$

Step 3. Determination of the characteristic frequency:

$$
\mathrm{F}=\frac{\mathrm{Y}_{1}(\mathrm{~N})}{\mathrm{Y}_{2}(\mathrm{~N})}
$$

Step 4. Finding the identification parameter characterizing the form of signal distribution according to the formula:

$$
\mathrm{A}=2 \cdot \mathrm{Y}_{2}(\mathrm{~N}) \cdot \mathrm{N} \text {. }
$$

Step 5. Identification of the identification number of distinguishable gradations (resolution)

$$
\text { Reg }=N / F .
$$


Step 6. Determination of the differences between the identification A-parameters of the investigated signal and the reference marks of the identification scale (IS) of the FRASHmethod - the matrix of communication of identification parameters with reference marks (Table I).

The reference marks are the names of symmetrical distributions (2mod - bimodal, asin - arcsine, even - uniform, trap - trapezoidal, simp - Simpson, gaus - normal, lapl Laplace, kosh - Cauchy). These names are taken from the vocabulary of the names of the distributions used for static measurements and, which, in this case, serve as the "digitized" reference marks of the IS.

TABLE I. THE IDENTIFICATION SCALE (IS) OF THE FRASH-METHOD

\begin{tabular}{|l|l|l|l|l|l|l|l|l|}
\hline $\begin{array}{c}\text { N=10000, } \\
\text { L=100 }\end{array}$ & \multicolumn{7}{|c|}{ Type of random signal distribution } \\
\cline { 2 - 9 } & 2mod & asin & even & trap & simp & gaus & Lapl & kosh \\
\hline Rank & 1 & 2 & 3 & 4 & 5 & 6 & 7 & 8 \\
\hline A, Rad & 4 & 6,28 & 8 & 10 & 12 & 20 & 36 & 3000 \\
\hline F, Hz & 2500 & 2027 & 1667 & 1380 & 1167 & 733 & 417 & 6 \\
\hline
\end{tabular}

Step 7. Ranking of differences by A-parameter in ascending order.

For example, if the test signal has $\mathrm{A}=30 \mathrm{Rad}$, then the difference in order of magnitude is as follows:

$\Delta 7=(\mathrm{ABS}(36$ (LAPL)-30) $=6 \mathrm{Rad}$,

$\Delta 6=(\mathrm{ABS}(20$ (GAUS) -30$)=10 \mathrm{Rad}$,

$\Delta 5=(\mathrm{ABS}(12$ (SIMP) -30$)=18 \mathrm{Rad}$,

$\Delta 4=(\mathrm{ABS}(10$ (TRAP) -30$)=20 \mathrm{Rad}$,

$\Delta 3=(\mathrm{ABS}(8(\mathrm{EVEN})-30)=22 \mathrm{Rad}$,

$\Delta 2=(\mathrm{ABS}(6,28(\mathrm{ASIN})-30)=23,72 \mathrm{Rad}$,

$\Delta 1=(\mathrm{ABS}(4(2 \mathrm{MOD})-30)=26 \mathrm{Rad}$,

$\Delta 8=(\mathrm{ABS}(3000(\mathrm{KOSH})-30)=2970 \mathrm{Rad}$.

Step 8. Formation of the positional code (PC) and the linguistic code (LC). In this case, the PC is a representation of the numbers of the standards of the ranked series, and the LC is a representation by name.

For example, if the investigated signal has $\mathrm{A}=30 \mathrm{Rad}$, then PC $=(76543218)$, and LC $=\{$ LAPL, GAUS, SIMP, TRAP, EVEN, ASIN, 2MOD, KOSH $\}$.

Step 9. Differences between the identification F-parameters of the investigated signal by standards and the IS reference marks of the FRASH method are determined.

Step 10. Ranking of differences by F-parameter in ascending order.

Step 11. Formation of PC and LC by F-parameter.

The results of identification measurements - PC and LC by A- and F-parameters.

Using the provisions of artificial intelligence and TISM, a block diagram of an intelligent computer device is presented in
Figure I. The device operates in two modes: "Linguistic Characteristics Output" and "LC Record" and includes two elements: a database (DB) and knowledge base (KB). The expert / operator is a specialist in the field of nondestructive testing and diagnostics, involved in measurement processes and the formation of linguistic assessments and conclusions recorded in the database.

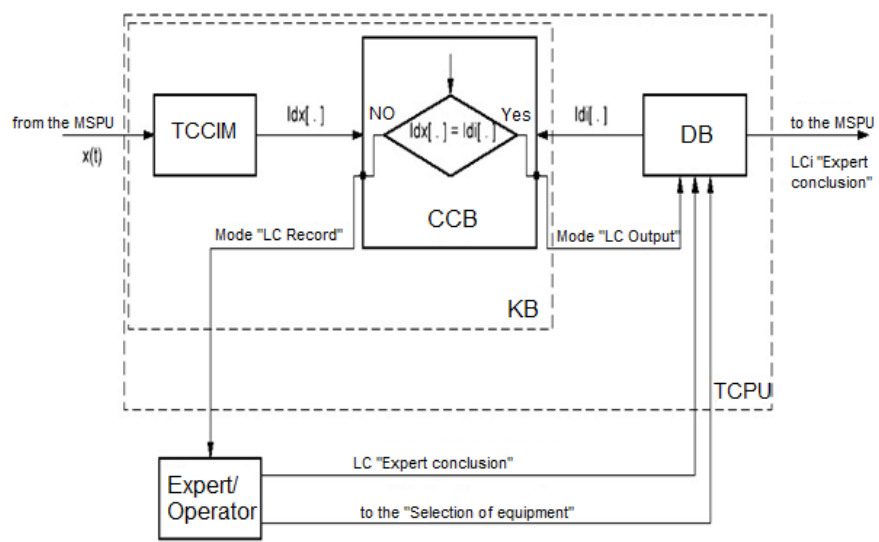

FIGURE I. STRUCTURAL DIAGRAM OF AN INTELLIGENT COMPUTER DEVICE (TIME PROCESSING UNIT)

The database has a structure in the form of a complex table II in the EXCEL environment, including positional and linguistic Idi[.] codes of the timing characteristics of the measurement signal and the corresponding linguistic expert estimates and conclusions ( $\mathrm{i}$ - the DB row number).

For each diagnostic object, the database (DB) is individual, therefore, before the measurement experiment, the operator generates a command "Selection of equipment."

TABLE II.DATABASE FORM

\begin{tabular}{|c|c|c|c|c|c|}
\hline \multicolumn{2}{|c|}{ Identification parameters, Idi[.] } & \multicolumn{3}{c|}{ Expert assessments and conclusions } \\
\hline Scale(X) & Scale(D) & $\ldots$ & IndMin(R) & $\begin{array}{l}<\text { Designation and } \\
\text { the defect name }>\end{array}$ & $\begin{array}{l}<\text { Recommendati } \\
\text { ons for removal }>\end{array}$ \\
\hline
\end{tabular}

In the BR, in accordance with the developed procedure, the generated time characteristic of the $\mathrm{X}(\mathrm{t})$ investigated signal is input from the output of the measuring signal processing unit (MSPU) to the time characteristic converter for identification measurements (TCCIM). An array of Idx [.] measurement parameters is formed at the output of the TCCIM.

In the cyclic comparison block (CCB), the corresponding positional and linguistic codes of the investigated signal are cyclically compared with those recorded in the database for each defective state. In the case of equality Idx [.] = Idi [.], the TCPU goes to the "LC output" mode and linguistic expert estimates and conclusions are output. Otherwise, the transition to the "Recording LC" mode and with the participation of an expert for Idx [.] linguistic characteristics are created with recording in the database.

The intelligent computer device is easily integrated into the "Korsar" diagnostic tool of the "ROS" SPE [6] used to diagnose oil and gas equipment, including rocking machines and pumps. The proposed modernized vibration analyzer 
performs not only traditional parametric and functional analysis, but also digital processing of time characteristics of measuring signals based on the proposed TISM methodology. In this case, the computer device, in agreement with the specialists of the "ROS" SPE was defined as a processing unit for the temporary characteristics of the diagnostic signal (the TCPU). The block diagram of the vibration analyzer with the TCPU is shown in Figure II.

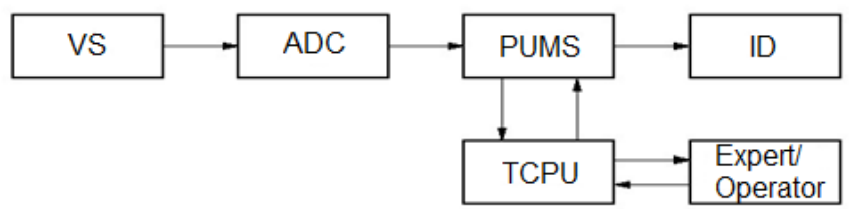

FIGURE II.

\section{BLOCK DIAGRAM OF THE VIBRATION ANALYZER WITH THE TCPU}

Figure II shows the blocks that are typical for existing vibro-analyzers: VS - vibration sensor, ADC - analog-digital converter, PUMS - processing unit for measuring signals, ID indicator device.

Block diagram of the algorithm developed by the software "IDS + Identification" is presented in Figure III.

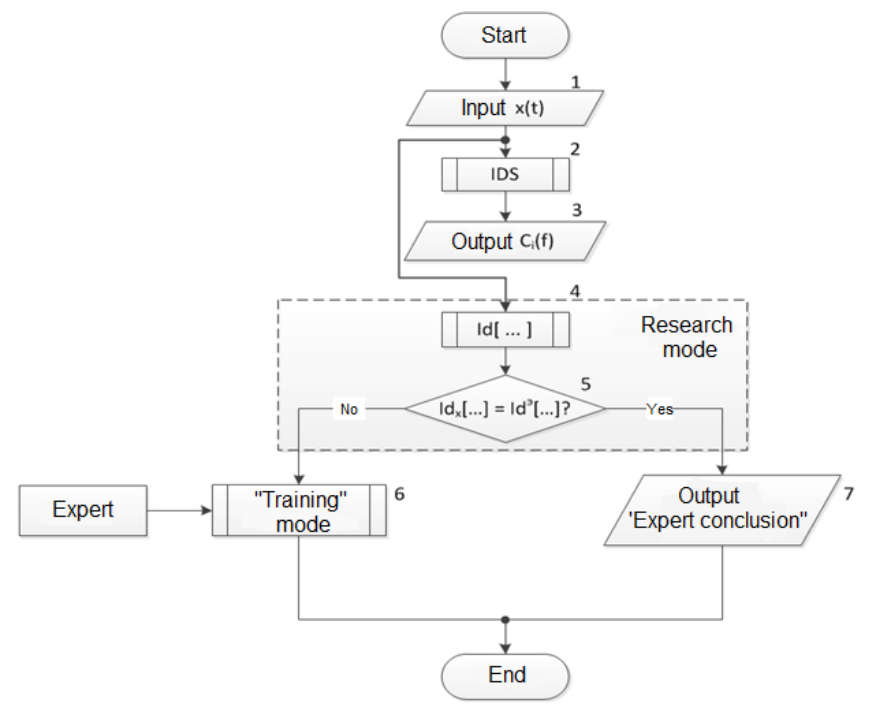

FIGURE III.

BLOCK DIAGRAM OF THE ALGORITHM OF THE SOFTWARE MODULE

The algorithm is implemented in the following stages.

Step 1. Input of the investigated vibrodiagnostic signal X (t) into the prototype of the "Kamerton" system with the "IDS + Identification" software.

Step 2. Signal processing in accordance with the existing "IDS" software, where it undergoes a Fourier series decomposition, and already in the frequency components $\mathrm{Ci}$ (f) - defective states (groups of defects with similar characteristics) are determined.

Step 3.Output of certain defective states Ci (f).
Step 4. Identification measurement of the $\mathrm{X}(\mathrm{t})$ signal using the methods described in Section 4, at the same time an Idx [...] array is formed at the output with identification parameters, names of the IS reference marks, position and linguistic codes. Their choice is determined by the selected designer and expert methods, when the database is formed.

Step 5. Identification of the defect by cyclic elementwise comparison of the Idx [...] array with reference Ide arrays [...] recorded in the database. In this case, the reference arrays are the values of the identification parameters, the names of the IS reference marks, the positional and linguistic codes of the defects recorded in the database according to the experts' conclusions.

If $\operatorname{Idx}[\ldots]=\operatorname{IdE}[\ldots]$, then go to Step 6, otherwise go to Step 7.

Step 6. Conclusion of the expert conclusion - the name of the defect identified name, recommendations for elimination, projected evaluation.

Step 7. Switch to the "Training" mode. In this case, the experts are studying specifically the object of research, a defect is established and an expert opinion is entered into the database on-line.

In accordance with the proposed method, the program module is integrated into the "IDS" software with the addition of a code identification function (Figure IV).

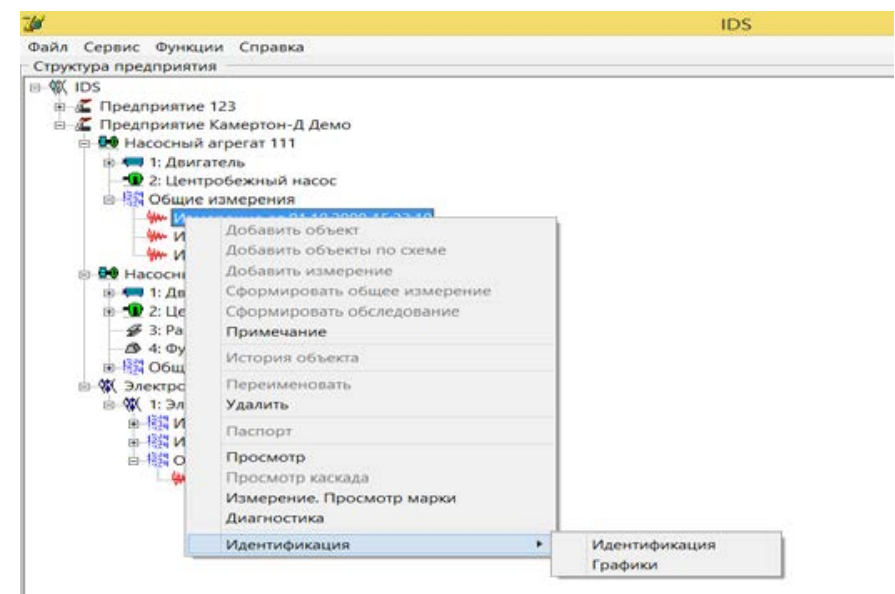

FIGURE IV. IDENTIFICATION (BY CODES) FROM THE GENERAL-PURPOSE CONTEXT MENU

In the "IDS + Identification" software there are two software submodules.

To enable the Identification function in the program, set the MsrIdentification parameter to 1 in the IDS.ini file in the ModesExtra section. Configure the authentication parameters through the MsrIdentification.conf file (Figure V).

The identification process is performed by the following actions. The call is made from the context menu of the general measurement of the unit, combining the measurements of vibration signals. Menu item - Identification - Measurement. 


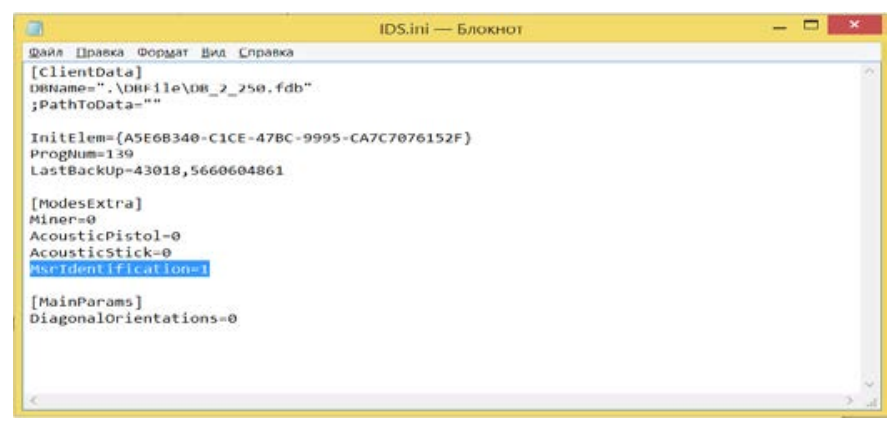

FIGURE V. SETTING OPTIONS

It is displayed in the window (Figure VI):

- expert's conclusion or automatic diagnosis depending on the regime;

- values of identification parameters at all points in the general measurement;

- type of equipment - a combination of equipment type code, unit diagram number and brand (for example, 304\$1\$HMA-8).

Available functions:

- he conclusion editing;

- copying parameters to the clipboard (Ctrl-C in the table with parameters);

- preservation of the identification measurement in the IM database (Save button).

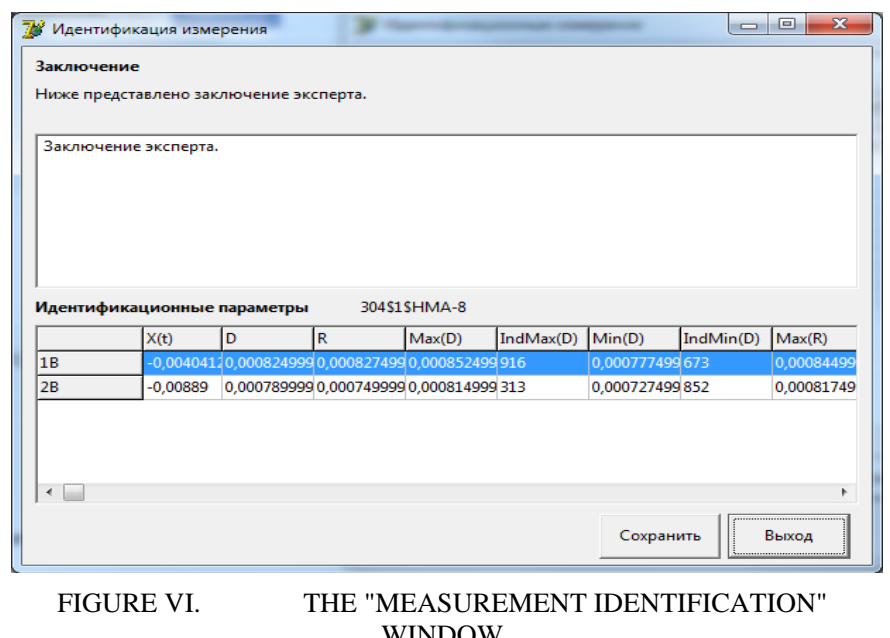

The operation of the "IDS + Identification" software module is based on the following sequence of the identification algorithm:

1) each point (signal) of the selected general measurement is analyzed;

2) the vibration signal is optionally converted to the selected units of measurement;

3) the first $\mathrm{N}$ samples are selected.
In the submodule, a set of identification parameters is calculated:

- the average value $\overline{\mathrm{X}(\mathrm{t})}$;

- identification parameters D, R, S, F, A, Reg;

- Max (D) is the maximum deviation of the trajectory (D);

- IndMax (D) is the coordinate of the maximum deviation of the trajectory (D);

- Min (D) is the minimum deviation of the trajectory (D);

- IndMin (D) - the coordinate of the minimum deviation of the trajectory (D);

- $\operatorname{Max}(\mathrm{R})$ is the maximum deviation of the trajectory (R);

- IndMax (R) is the coordinate of the maximum deviation of the trajectory $(\mathrm{R})$;

- $\operatorname{Min}(\mathrm{R})$ is the minimum deviation of the trajectory (R);

- IndMin (R) is the coordinate of the minimum deviation of the trajectory $(\mathrm{R})$.

The "IDS + Identification" software was installed on the prototype of the "Kamerton" computer device, presented in Figure VII.

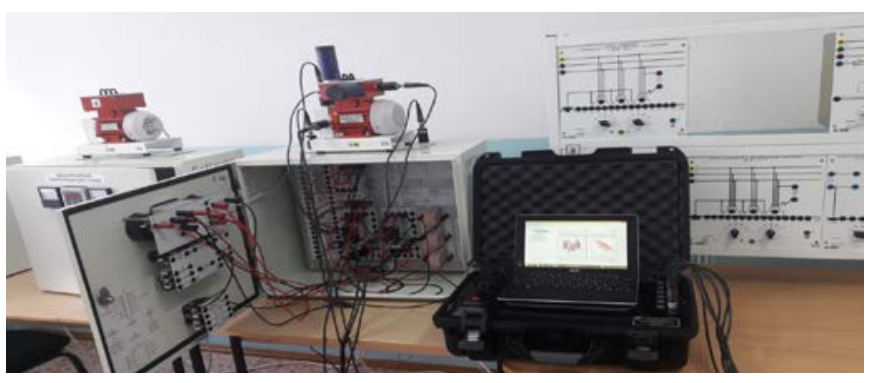

FIGURE VII.

PROTOTYPE OF A COMPUTER DEVICE

\section{RESUlts}

1) the TISM elements can be used to solve the problems of assessing the qualitative state of dynamic diagnostic objects, since on average $95 \%$ probability of recognizing certain defects of the pumping unit: CD.Mm2.0 - "Mechanism imbalance", CD.Pr.2.0 - "Increased clearances in the bearing ", CD.Mm.9.0 -" Bending the motor shaft ";

2) the developed intelligent technologies for recognition of vibration signals and watt-metro-gram allowed to recognize with 95\% probability 6 defects of the rocking machines: JPD.JSR.2.0 Defect of the jack shaft of the 2 reducer, JPD.GDDSJS.10 - the defect of the gear drive "drive shaft - 1 jack shaft" gearbox, JPD.GDJSJS.1.2 - Defect of the gear drive "jack shaft 1 - jack shaft 2" of the reducer, JPD.GDJSDS.2.0 - defect of the gear drive "2 jack shaft driven shaft" reducer, JPD.HFPP.1.0 - high fit plunger pump,JPD.LFPP.1.0 - low fit of pump plunger; 
3) it is convenient to measure the properties of the regularity-randomness of vibration signals with the help of the 3 scales technology: two fixed measuring ranges (D-Scale, RScale), and the third M-Scale measuring determining during the scanning phase the distance of travel and its position relative to D-Scale and R-Scale;

4) the regularity-randomness degree is determined by the relative distance between the measuring and two other scales, and if the M-Scale after scanning is located exactly in the middle between the scales (D-Scale, R-Scale), it means that the analyzed signal is $100 \%$ chaotic (unordered);

5) the shift of the measuring scale from the center, in one direction or another, indicates the presence of some regularity (order) in the signal, since D-Scale and R-Scale are ordered, respectively, in increasing and decreasing values of samples of the analyzed signal;

6) the presence of some regularity (order) in the signal indicates, with a certain probability, the presence of signs of defects in the object of diagnosis.

7) the correspondence between the results of the identification measurements of the degree of regularityrandomness and the types of possible defects is established by logical analysis with the knowledge of experts of the given subject area, and therefore, this procedure is individual for each analyzed object;

8) the intelligent block of the TCPU is fully implemented with the use of modern microcontrollers, for example STM32, and is easily integrated into modern vibro-analyzers;

9) the compact "KORSAR" vibrometer of the "ROS" company (Perm city), modernized by the integration of the TCPU, thanks to its small dimensions and ease of use, successfully and effectively measured vibration, stored the results of measurements in the memory of the vibrometer, conducted initial processing using the method of identification measurements , transmitted via USB and RS-232 interfaces to the computer for processing in the "IDS + Identification" program.

Consequently, the use of artificial intelligence technologies and scientific methods of analyzing the TISM on the basis of time characteristics makes it possible to perform diagnostics and monitoring by switching from recognition of states to identification of individual defects, since each defect creates individual forms of signal characteristics.

The direction of further research should be concentrated on building up a database of computer devices for identification measurements to identify other defects in oil and gas equipment operating in industrial enterprises, through the collection of expert opinions using Internet technologies.

A distinctive feature of the intelligent monitoring and diagnostic system is the ability to recognize not only the state of objects, but also to identify the defects themselves, which was confirmed experimentally.

The availability of a multichannel data collection system makes it reasonable to use wireless modern infocommunication technologies for data transmission, the implementation of standards widely used in industry made it possible to provide increased functionality in the collection and information transmission from sensors.

The computer device proposed by the authors has a great perspective, in particular, in the automation and management of oil and gas production processes ("Smart Well"), efficient transportation with energy-saving regimes, and maintenance of complex explosive processing equipment.

In the future, the possibility of using the developed computer devices for diagnostics of equipment in related industries is considered: electric power stations and substations, seismology, biomedicine, etc.

\section{REFERENCES}

[1] Barkov A.V., Barkova N.A. Vibration diagnosis of machinery and equipment. Analysis of vibration: Study Guide. - SPb .: SPbGMTU, 2004. - 176 p.

[2] Dyakonov V.P. Wavelets. From theory to practice. 2nd edition. M .: SOLON-Press, 2010. - 400 p.

[3] Pavlov S.N. Systems of artificial intelligence. M .: El Content, 2011. 174 p.

[4] Koshekov K.T., Klikushin Yu.N. Theoretical basis of identification measurements and signal transformations. Monograph. - Saarbrucken, Germany: LAP LAMBERT Academic Publishing GmbH \& Co.KG, 2011. - 171 p.

[5] Klikushin Yu. N., K. T. Koshekov, V. Yu. Kobenko, E. S. Trunin An algorithm for evaluating the state of a vibrosignals. Russian Journal of Nondestructive Testing July 2014, Volume 50, Issue 7, 413-418.

[6] http://www.ros-diagnostics.ru. 